$\left.\begin{array}{c}\text { Kalpa Publications in Engineering } \\ \text { Volume 3, 2020, Pages 74-78 } \\ \text { Proceedings of International Sym- } \\ \text { posium on Applied Science 2019 }\end{array}\right)$ Kalpel

\title{
DFT Study On Adsorption Of Volatile Organic Compounds On Silicene
}

\author{
Trong Lam Pham ${ }^{1}$, Van On Vo ${ }^{2}$, Van An Dinh* ${ }^{1,3}$ \\ ${ }^{1}$ Nanotechnology Program, VNU Vietnam Japan University, My Dinh Campus, Nam Tu Liem, Ha \\ Noi, Vietnam \\ ${ }^{2}$ Group of Computational Physics and Simulation of Advanced Materials - Faculty of Natural \\ Sciences - University of Thu Dau Mot, Vietnam \\ ${ }^{3}$ Center for Atomic and Molecular Technologies, Graduate School of Engineering, Osaka \\ University, Yamadaoka 2-1, Suita, Osaka 565-0871, Japan \\ *Corresponding author's email: dv.an@vju.ac.vn
}

\begin{abstract}
Cancer can be regarded as a rising threat towards modern societies. Detecting cancer at an early stage significantly improves the curability of the disease; unfortunately, currently available methods for early diagnosis of cancer are scarce and inefficient. In fact, the concentration of Volatile Organic Compounds (VOCs) in cancer patients in the breath is different from that in normal people. Therefore, development of new sensors that can detect VOCs with low concentrations at the early stage of cancer, is desirable. 2D materials are expected as attractive materials for these sensors due to their large surface area to volume ratio. In this work, we investigated the adsorption mechanism of some small-to-medium VOCs on the surface of silicene by the quantum simulation method. The images of the potential energy surfaces for different positions of the adsorbate on the silicene surface were explored by Computational DFT-based Nanoscope for determination of the most stable configurations and diffusion possibilities. The adsorption energy profiles were calculated by three approximations of van der Waals interation: revPBE-vdW, optPBE-vdW, and vdW-DF2. It is found that the adsorption energies of the VOCs in question vary in the range of $0.6-1.0 \mathrm{eV}$, which indicates that silicene is considerably sensitive with these VOCs. The charge transfer between the substrate and VOCs was also addressed.
\end{abstract}

Keywords: Volatile Organic Compound, Adsorption, Silicene, DFT, Cancer Detection 


\section{Introduction}

It is known that some Volatile Organic Compounds (VOCs) exist in the breath of cancer patient at different concentrations than in healthy people. In addition, their concentration differences depend on the type of cancer [1,2]. As a result, measuring VOCs' concentration is expected to be a promising non-invasive cancer diagnosis. Unfortunately, their super-low concentration is challenging us in desiging a highly sensitive devices for detecting VOCs. The search for appropriate materials to fabricate VOC sensors became glowing. Among the potential candidates, 2D materials are attracting the great attention from scientists since the discovery of graphene. Currently, research focusing on the potential of 2D materials to be used in VOC sensors is lacking. Here we explore the possibility for use of the free-standing silicene [3] (an analog of graphene) as a material for VOC sensors by theoretical investigation.

\section{Methods}

All calculations were done using the software package Vienna Ab initio Simulation Package (VASP). Different correction schemes were considered to account for van der Waals interactions (revPBE-vdW [4], optB88-vdW, optB86b-vdW, optPBE-vdW [5,6], and vdW-DF2). Three VOCs (Acetone, Trichloroethene, and Toluene) belonging to the three different chemical functional groups (ketones, halides, and aromatics) were selected to represent VOCs existing in the breath of cancer patients. Their adsorption mechanisms on the surface of silicene $4 \times 4$ supercell (Figure 1) were investigated by Density Functional Theory (DFT). The most favorable position and configuration of the adsorbate on the silicene substrate were determined by performing automatic scanning with Computational DFT-based Nanoscope [7].
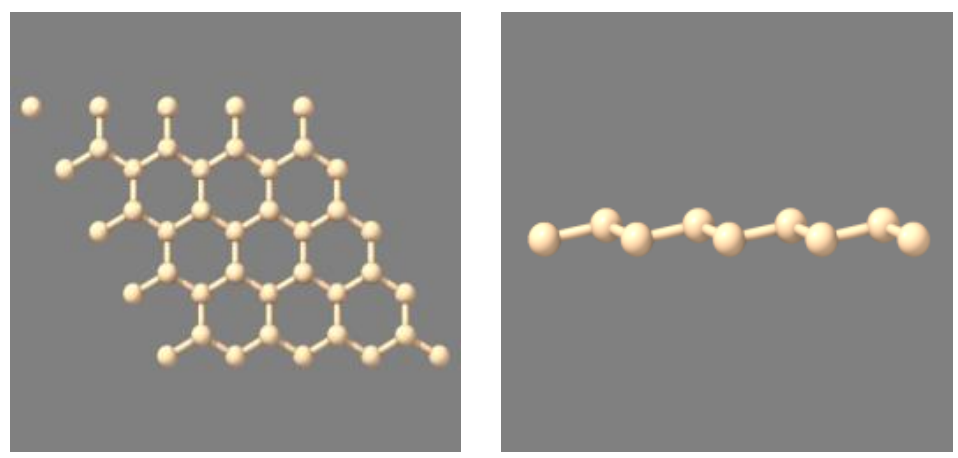

Figure 1: Free-standing silicene $4 \times 4$ supercell model

\section{Results and Discussion}

\subsection{Stable position and Diffusion Possibility}

The stable position of adsorbates can be deduced from the minima on the Potential Energy Surface (PES) as shown in Fig. 2. On the left are 3D PESs, on the right are 2D projected PESs superimposed 
onto the silicene supercell. Red circles represent upper layer silicon atoms, while transparent ones demonstrate atoms on the lower Si layer. The color gradient illustrates the relative interaction energy: the darker color area is more preferable for adsoption. The possible diffusion pathways are shown by yellow solid lines.
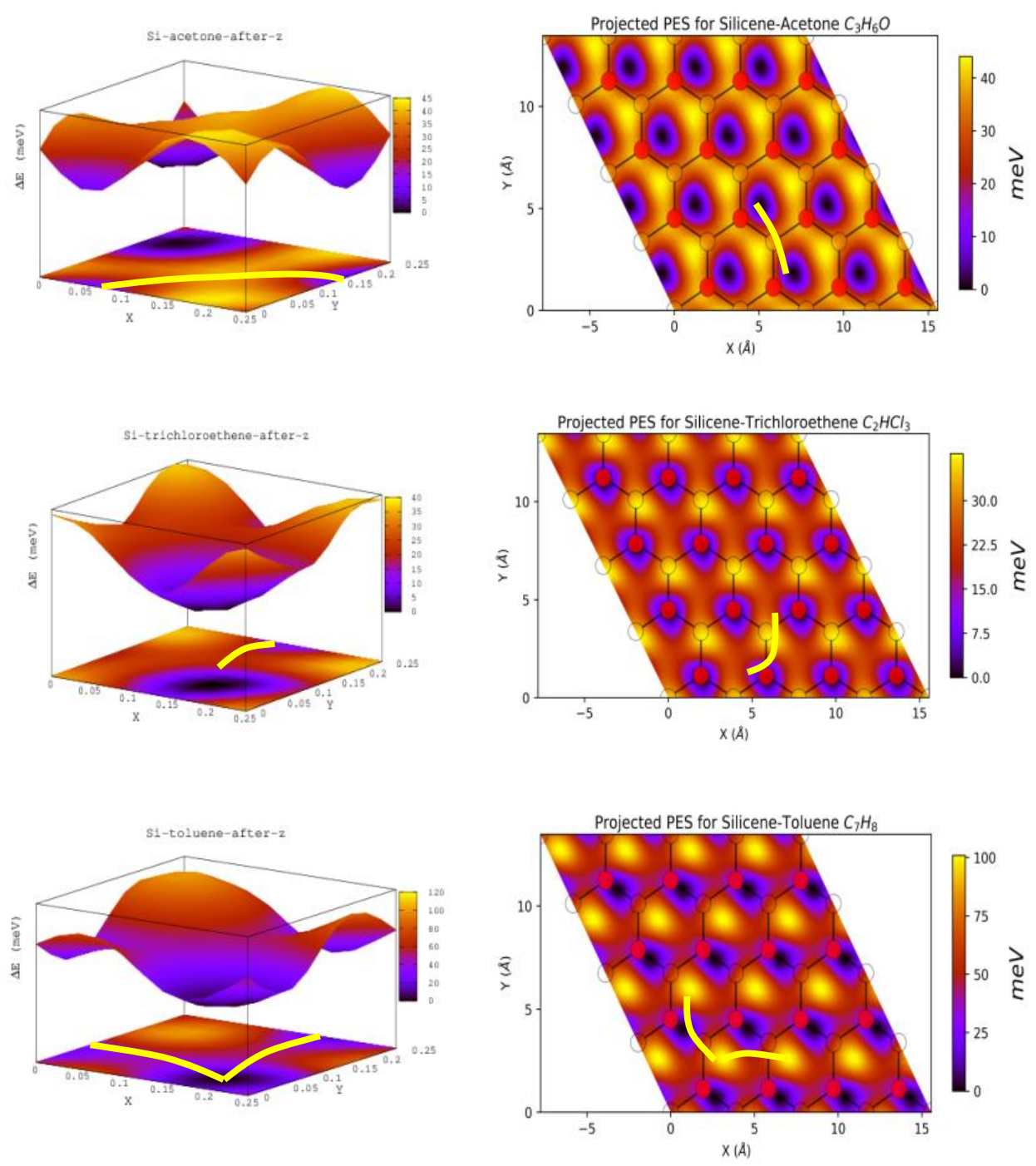

Figure 2: Projected PES for VOCs on silicene. Left: 3D PES. Right: Projected PES. Top to bottom: Acetone, Trichloroethene, Toluene adsorption on silicene.

For acetone, the PES minima lies at the middle of the bond connecting two lower-layer atoms; while trichloroethene prefers to be on top of the upper layer atoms, and toluene at the middle of the bond connecting one upper and one lower atom. Different substrates display different possible pathways for diffusion. The corresponding diffusion barriers are estimated to be $\sim 20, \sim 25$, and $\sim 50$ $\mathrm{meV}$, respectively for acetone, trichloroethene, and toluene. This difference in diffusion barrier might affect the sensor response. In order to detect the change in the electric current signal, the change in the 
resistance should be sufficient. This change is determined by varying adsorbate concentration. The first VOCs molecules will occupy the locations where most accessible. If the adsorbed VOCs are difficult to diffuse to other locations, the next VOC adsorption might be hindered, which lengthens the time required to achieve sufficient resistance change. Therefore, this diffusion barrier trend suggests that the response of Silicence towards toluene is slower than towards acetone and trichloroethene.

\subsection{Adsorption Profile}

The adsorption profiles for different VOCs are shown in Fig.3. The x-axis represents the distance from the center of mass (COM) of the VOC to the silicene surface. The y-axis corresponds to the adsorption energy, which is defined as:

$$
E_{\text {adsorption }}=E_{\text {complex }}-E_{\text {saturation }}
$$

in which $E_{\text {complex }}$ is the total energy of the system (adsorbate + substrate), $E_{\text {saturation }}$ is the total energy of the system at which there is no interaction between VOC and silicene.

Initially, we employed five different van der Waals functionals; however, as can be seen in the adsorption profile of silicene-acetone system, optB88-vdW and optB66b-vdW gave almost identical results with optPBE-vdW. Therefore, for other VOCs, we performed calculations with three schemes (revPBE-vdW, optPBE-vdW, and vdW-DF2).
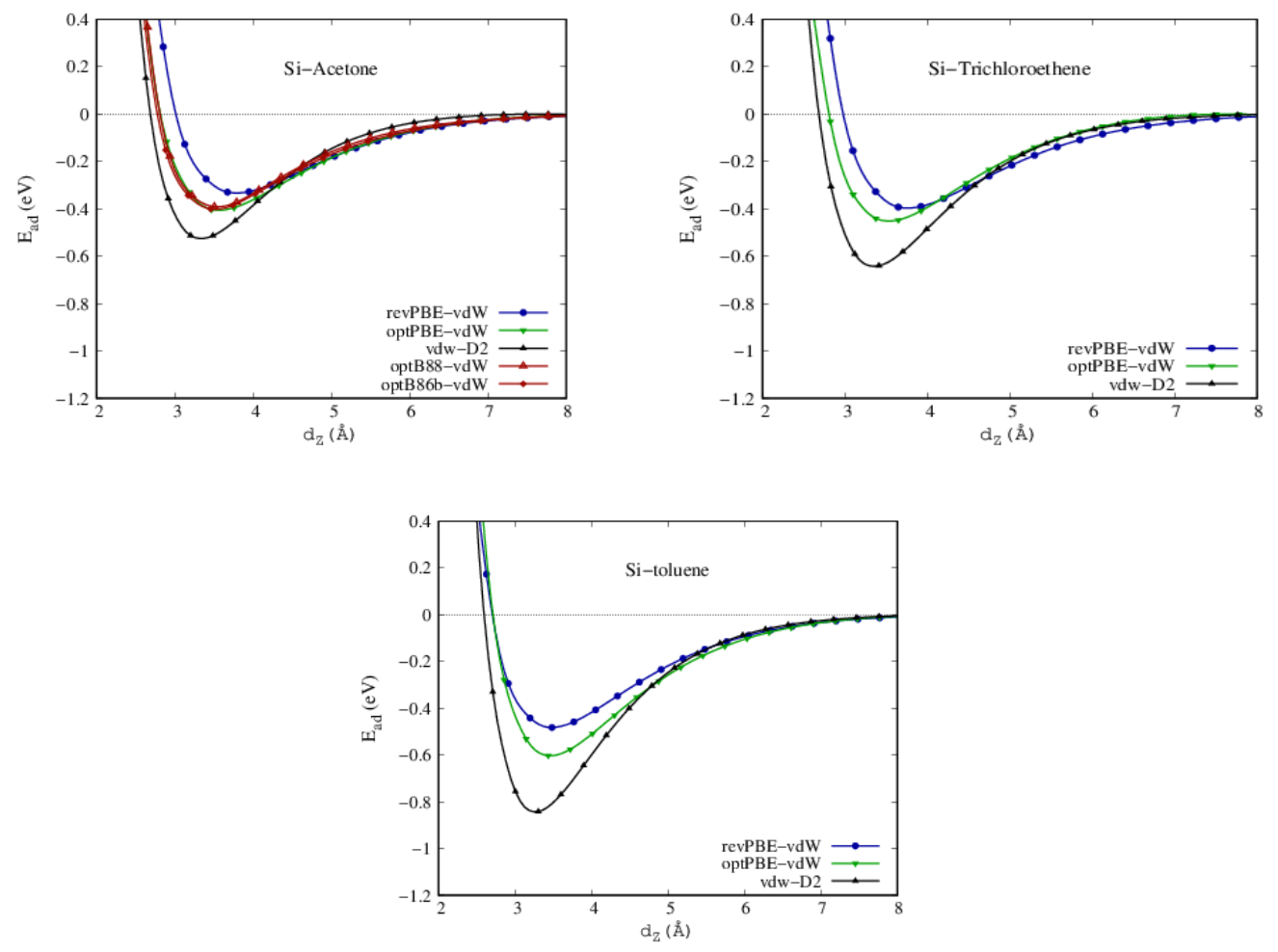

Figure 3: Adsorption Profile for VOCs on silicene 
Three functionals provide the estimation range for the adsorption characteristics. Although the distances from the COM of VOCs to the silicene surface are similar, the differences of distances from the closest atom in VOCs to the silicene surface, and the adsorption energies allow us to draw some interesting remarks. revPBE-vdW gave much larger distance and lower adsorption energy, which can give a rough estimation about the upper bound for the distance and the lower bound for the adsorption energy. Acetone adsorption has the smallest adsorption energy, although acetone comes close to the silicene surface $(\sim 2.7 \AA)$. For Trichlorothene, although the molecule stays at a much larger distance $(\sim 3.4 \AA)$, a slightly larger adsoprtion energy was obtained. On the contrary, adsorption of toluene requires a largest energy and the molecules also can come closest to the surface $(\sim 2.6 \AA)$. This indicates strong interaction between toluene and silicene surface, which might be explained in terms of partial overlap between the conjugated $p$ orbitals of carbon in the aromatic ring with the $p_{z}$ orbitals of silicon. Note that although free-standing silicene is buckled, the $\mathrm{sp}^{2}$ characteristic is more dominant than the $\mathrm{sp}^{3}$ characteristic (in terms of bond length). The strong interaction between toluene and silicene suggests the largest charge transfer, and in effect, largest change in resistance of the silicene. In turn, this implies that silicene is more sensitive toward toluene.

\section{Conclusions}

The adsorption energies suggest that silicene is more sensitive toward toluene than toward acetone and trichloroethene. Therefore, silicene can be expected to have good sensitivity for aromatic compounds due to the strong interaction. From the large adsorption energies towards VOCs, silicene can be expected to be a promising material for VOC sensing.

\section{References}

[1] M. Hakim, Y.Y. Broza, O. Barash, N. Peled, M. Phillips, A. Amann, H. Haick, Volatile Organic Compounds of Lung Cancer and Possible Biochemical Pathways, Chem. Rev. 112 (2012) 5949-5966. doi:10.1021/cr300174a.

[2] E.M. Gaspar, A.F. Lucena, J. Duro da Costa, H. Chaves das Neves, Organic metabolites in exhaled human breath-A multivariate approach for identification of biomarkers in lung disorders, J. Chromatogr. A. 1216 (2009) 2749-2756. doi:10.1016/j.chroma.2008.10.125.

[3] P. Vogt, G. Le Lay, G. (Guy) Le Lay, Silicene: Prediction, Synthesis, Application, n.d.

[4] M. Dion, H. Rydberg, E. Schröder, D.C. Langreth, B.I. Lundqvist, Van der Waals Density Functional for General Geometries, Phys. Rev. Lett. 92 (2004) 246401. doi:10.1103/PhysRevLett.92.246401.

[5] J. Klimeš, D.R. Bowler, A. Michaelides, Chemical accuracy for the van der Waals density functional, J. Phys. Condens. Matter. 22 (2010) 0-5. doi:10.1088/0953-8984/22/2/022201.

[6] J. Klimeš, D.R. Bowler, A. Michaelides, Van der Waals density functionals applied to solids, Phys. Rev. B. 83 (2011) 195131. doi:10.1103/PhysRevB.83.195131.

[7] V. A. Dinh, Computational DFT-based Nanoscope -- a tool for exploring the adsorption of molecules on the surface of 2D materials, (2018). 